\title{
REGULATING HULLS OF ALMOST COMPLETELY DECOMPOSABLE GROUPS
}

\author{
A. MADER and C. VINSONHALER
}

(Received 6 May 1991; revised 25 July 1991)

Communicated by P. Schultz

\begin{abstract}
This note investigates torsion-free abelian groups $G$ of finite rank which embed, as subgroups of finite index, in a finite direct sum $C$ of subgroups of the additive group of rational numbers. Specifically, we examine the relationship between $G$ and $C$ when the index of $G$ in $C$ is minimal. Some properties of Warfield duality are developed and used (in the case that $G$ is locally free) to relate our results to earlier ones by Burkhardt and Lady.
\end{abstract}

1991 Mathematics subject classification (Amer. Math. Soc.): $20 \mathrm{~K} 15$.

\section{Introduction}

In 1937, R. Baer [4] gave a promising start to the theory of torsion-free abelian groups of finite rank with his classification of the completely decomposable groups, those groups isomorphic to a direct sum of subgroups of the additive rationals, $Q$. An obvious way in which to try to extend Baer's results is to look at the almost completely decomposable groups-those torsion-free abelian groups of finite rank which contain a completely decomposable group as a subgroup of finite index. Given the success of Baer, it is perhaps surprising that even with the finite rank assumption, almost completely decomposable groups have proved so intractable. In 1979, L. Lady [7] defined and studied the regulating subgroups of an almost completely decomposable group $G$ : those completely decomposable subgroups of $G$ of minimal index in

The research of the second author was supported in part by NSF Grant DMS-9022730.

(C) 1993 Australian Mathematical Society 0263-6115/93 \$A2.00+0.00 
$G$ among all completely decomposable subgroups. A discouraging fact, illustrated by Lady, is that if $C$ is a regulating subgroup of $G$, the finite quotient $G / C$ need not be an invariant of $G$. That is, different regulating subgroups can give non-isomorphic quotients. Five years later, Burkhardt [5] defined the regulator of an almost completely decomposable group as the intersection of all regulating subgroups. The regulator is a completely decomposable fully invariant subgroup of finite index, a fact, apparently folklore, which was used to study isomorphism of almost completely decomposable groups in [6]. Until recently, the three papers discussed above represented most of the work on almost completely decomposable groups (see references).

The goal of this note is a "dualization" of the basic facts on regulating subgroups and regulators established by Lady [7] and Burkhardt [5]. Instead of treating completely decomposable subgroups of finite index in a given group $G$, we consider completely decomposable groups which contain $G$ as a subgroup of finite index. Our methods shed some additional light on the results in [7] and [5]. In the locally free setting, Warfield duality can be applied to show directly the equivalence of our results to those of Lady and Burkhardt (Section 2). The duality relationship in the general (not locally free) setting remains an intriguing mystery. In any case, we consider it worthwhile to give a cohesive treatment of the fundamental relationships between a completely decomposable group and other groups in its quasi-equality class. The hope is that our "dual" results can be combined with the known results on regulating subgroups and regulators to facilitate further understanding of a complicated class of groups.

\section{Regulating Hulls}

In what follows, all groups are abelian and are torsion-free finite rank unless they are finite quotients of such. We regard each torsion-free group $G$ as a subgroup of its divisible hull $Q G$, a $Q$-vector space. Other standard notation and terminology follow [7] and [2]. In particular, subgroups $G$ and $H$ of the same $Q$-vector space are called quasi-equal if there is a positive integer $n$ such that $n G \subseteq H$ and $n H \subseteq G$. More generally, two torsion-free groups $G$ and $H$ are quasi-isomorphic if $G$ is isomorphic to a subgroup of finite index in $H$.

A male $A C D$-pair is an embedding $G \rightarrow C$ where $C$ is completely decomposable of finite rank and the image of $G$ has finite index in $C$. The index of the image of $G$ in $C$ will be called the index of the $A C D$-pair. This index is said to be minimal if it is minimal among all indices obtained by imbedding $G$ in a completely decomposable group. A female $A C D$-pair 
is an embedding $C \rightarrow G$, with index defined analogously. The terms "male" and "female" will generally be omitted where there is no chance of confusion. A type is an isomorphism class of rank one groups. We will write $C=\bigoplus C_{\sigma}$, where each $C_{\sigma}$ is a homogeneous completely decomposable group of type $\sigma$. In the interest of brevity, we write $C_{\leq \sigma}=\bigoplus_{\tau \leq \sigma} C_{\tau}$ and $C_{<\sigma}=\bigoplus_{\tau<\sigma} C_{\tau}$. The set of types for which $C_{\sigma} \neq 0$ is called the critical typeset of $C$ (or $G)$. Whenever a type $\tau$ or $\sigma$ is mentioned, it tacitly is understood to be in the critical typeset; and unless otherwise indicated, summations are over the critical typeset.

A Butler group is a pure subgroup of a finite rank completely decomposable group. Because the class of Butler groups is closed under quasi-isomorphism (see [1]), almost completely decomposable groups are Butler groups and we have the extensive machinery from that class at our disposal ([1] and [2]). In the first part of the paper, we work primarily with homomorphic images of almost completely decomposable groups $G$, and employ extensively the following functorial subgroups.

$$
G[\sigma]=\langle x \in G \mid \operatorname{type}(x) \not \sigma\rangle_{*}=\bigcap\{\operatorname{Ker} f \mid f: G \rightarrow Q, \text { type } f(G) \leq \sigma\}
$$

and

$$
G^{*}[\sigma]=\bigcap_{\tau<\sigma} G[\tau]
$$

We will use, frequently and without reference, the fact that (for any Butler group $G$ )

$$
0 \rightarrow G^{*}[\sigma] / G[\sigma] \rightarrow G / G[\sigma] \rightarrow G / G^{*}[\sigma] \rightarrow 0
$$

is a split exact sequence of groups, with $G^{*}[\sigma] / G[\sigma]$ homogeneous completely decomposable of type $\sigma$ (see [2]). Any map $G / G[\sigma] \rightarrow G^{*}[\sigma] / G[\sigma]$ which splits this sequence will be called a splitting map.

Proposition 1.1. Let $G \subset C=\bigoplus C_{\sigma}$ be an $A C D$-pair and let $\sigma$ be a critical type.

(a) There is a commutative diagram with exact rows,

$$
\begin{aligned}
& 0 \rightarrow C[\sigma] \rightarrow C \rightarrow C_{\leq \sigma} \rightarrow 0 \\
& 1 \uparrow \rightarrow \begin{array}{l}
1 \uparrow \\
0 \rightarrow G[\sigma] \rightarrow G
\end{array} \rightarrow G / G[\sigma] \rightarrow 0
\end{aligned}
$$

where $\alpha_{\sigma}$ is the monomorphism induced by projection onto $C_{\leq \sigma}$.

(b) There is a commutative diagram with exact rows,

$$
\begin{array}{ccccccc}
0 \rightarrow & C_{\sigma} & \rightarrow & C_{\leq \sigma} & \rightarrow & C_{<\sigma} & \rightarrow 0 \\
& \alpha_{\sigma}^{\prime} \uparrow & & \alpha_{\sigma} \uparrow & & \beta_{\sigma} \uparrow & \\
0 \rightarrow & G^{*}[\sigma] / G[\sigma] & \rightarrow & G / G[\sigma] & \rightarrow & G / G^{*}[\sigma] & \rightarrow 0
\end{array}
$$


where $\alpha_{\sigma}^{\prime}: G^{*}[\sigma] / G[\sigma] \rightarrow C_{\sigma}$ is the restriction of $\alpha_{\sigma}: G / G[\sigma] \rightarrow C_{\leq \sigma}$, and $\beta_{\sigma}$ is the monomorphism induced by projection onto $C_{<\sigma}$.

Moreover, in both (a) and (b), the induced sequence of cokernels is exact.

Proof. Routine exercise.

We will denote by $\bar{G}_{\sigma}, \bar{G}=\bar{G}_{\leq \sigma}$, and $\bar{G}_{<\sigma}$ the images of the three maps $\alpha_{\sigma}^{\prime}, \alpha_{\sigma}$ and $\beta_{\sigma}$ in 1.1(b).

Proposition 1.2. Let $G$ be an almost completely decomposable group. For any choice of splitting maps $\pi_{\sigma}: G / G[\sigma] \rightarrow G^{*}[\sigma] / G[\sigma]$, the composition $G \rightarrow \bigoplus G / G[\sigma] \rightarrow \bigoplus G^{*}[\sigma] / G[\sigma]$ defines an ACD-pair. The index of this pair is independent of the choice of the $\pi_{\sigma}$ 's.

Proof. Let $G \rightarrow C=\bigoplus C_{\sigma}$ be an $A C D$-pair. Denote $D_{\sigma}=G^{*}[\sigma] / G[\sigma]$ and $D=\oplus D_{\sigma}$. The composition $\pi: G \rightarrow \bigoplus G / G[\sigma] \stackrel{\oplus \pi_{\sigma}}{\longrightarrow} \oplus D_{\sigma}$ is a monomorphism (for any Butler group $G$ ) by [2], Corollary 1.8(b). Since $G$ is quasi-equal to $C, D_{\sigma}$ is isomorphic to $C_{\sigma}$, as both are homogeneous completely decomposable and (quasi-)isomorphic to $G^{*}[\sigma] / G[\sigma]$, noting that quasi-isomorphic homogeneous completely decomposable groups are isomorphic (see [1]). It follows that $G$ is quasi-isomorphic to $D$ and the index of $\pi(G)$ in $D$ is finite.

To show the index is independent of the choice of the $\pi_{\sigma}$ 's, it suffices to fix $\tau$ and assume $\left\{\pi_{\sigma}^{\prime}\right\}$ are splitting maps with $\pi_{\tau} \neq \pi_{\tau}^{\prime}, \pi_{\sigma}=\pi_{\sigma}^{\prime}$ for $\sigma \neq \tau$. Let $\pi^{\prime}: G \rightarrow D$ be the $A C D$-pair defined by replacing $\bigoplus \pi_{\sigma}$ with $\bigoplus \pi_{\sigma}^{\prime}$. First assume $G[\tau] \neq 0$. Note that $\pi(G[\tau])=\pi^{\prime}(G[\tau])$. Moreover, both $\pi$ and $\pi^{\prime}$ induce embeddings $G / G[\tau] \rightarrow \bigoplus_{\sigma \leq \tau} G / G[\sigma] \rightarrow D_{\leq \tau}$. Since there is a canonical isomorphism $(G / G[\tau]) /(G / G[\tau])[\sigma] \simeq G / G[\sigma]$ for $\sigma \leq \tau$, we can apply induction on the rank of $G$ to conclude that the imbeddings $G / G[\tau] \rightarrow D_{\leq \tau}$ induced by $\pi$ and $\pi^{\prime}$ have the same index. An appeal to Proposition 1.1(a) now shows that the original embeddings $\pi$ and $\pi^{\prime}$ have the same index.

We conclude the proof by treating the case where $G[\tau]=0$. In this case, $G=G^{*}[\tau] \oplus H$, where $H \simeq G / G^{*}[\tau]$. Furthermore, $\pi$ and $\pi^{\prime}$ induce embeddings $G / G^{*}[\tau] \rightarrow \bigoplus_{\sigma<\tau} G / G[\sigma] \rightarrow D_{<\tau}$ whose index is the same as that of the original embeddings. This is because $D_{\tau}=G^{*}[\tau]$ is a summand of $G$. Since the rank of $G / G^{*}[\tau]$ is less than the rank of $G$, we may again apply induction to obtain the equality of the two indices.

We call the index defined by Proposition 1.2 the male index of $G$ and denote it by $\mu(G)$. 
LEMMA 1.3. Let $G \subseteq H_{1} \oplus H_{2}$ be an $A C D$-pair, and $G_{1}$ the projection of $G$ onto $H_{1}$. If $l: G_{1} \rightarrow H^{\prime}$ is an $A C D$-embedding with finite index $k$, then there is a subgroup $H_{1}^{\prime}$ of $Q H_{1}$ such that $G_{1} \subseteq H_{1}^{\prime} \simeq H^{\prime}$ and $\left|H_{1}^{\prime} / G_{1}\right|=k$. Furthermore, $G \subseteq H_{1}^{\prime} \oplus H_{2}$ is an $A C D$-pair.

Proof. Note that $l$ extends uniquely to a vector space map $Q G_{1} \rightarrow Q H^{\prime}$. Then $H_{1}^{\prime}$ is just the inverse image of $H^{\prime}$ (in $Q G_{1}=Q H_{1}$ ) under this extension map.

This lemma will be utilized to replace a component $C_{\leq \sigma}$ in an $A C D$-pair $G \rightarrow C=C_{\leq \sigma} \oplus C[\sigma]$ by a quasi-equal component $C_{\leq \sigma}^{\prime}$.

Proposition 1.4. Let $G \subseteq C=\bigoplus C_{\sigma}$ be an $A C D$-pair of minimal index and $\sigma$ a critical type. Then, in the notation of Proposition 1.1,

(a) $G / G[\sigma] \stackrel{\alpha_{a}}{\rightarrow} C_{\leq \sigma}$ and

(b) $G / G^{*}[\sigma] \stackrel{\beta_{a}}{\rightarrow} C_{<\sigma}$

are ACD-pairs of minimal index. Moreover, the indices in (a) and (b) are equal.

Proof. It is routine that the two embeddings are $A C D$-pairs.

Let the index in (a) be $k$. To see that $k$ is minimal, suppose that $G / G[\sigma] \rightarrow D_{0}$ is an $A C D$-pair of index $k_{0}$. By applying Lemma 1.3 to $G \subseteq C_{\leq \sigma} \oplus C[\sigma]$, we can assume $Q D_{0}=Q C_{<\sigma}$ and $G \subset D_{0} \oplus C[\sigma]=D$ is an $A C D$-pair. By Proposition $1.1(\mathrm{a}),|D / G|=|C[\sigma] / G[\sigma]| \cdot k_{0}$ and $|C / G|=|C[\sigma] / G[\sigma]| \cdot k$. Since $|C / G|$ is minimal, we have $k \leq k_{0}$ and $k$ is minimal.

The proof of (b) is similar, using that the embedding in (a) has minimal index. Let the index in (b) be $j$ and suppose $G / G^{*}[\sigma] \rightarrow D_{1}$ is an $A C D$-pair of index $j_{0}$. By Lemma 1.3, we may assume that $Q D_{1}=Q C_{<\sigma}$ and that $G / G[\sigma] \rightarrow C_{\sigma} \oplus D_{1}$ is an $A C D$-pair. By Proposition 1.1(b), the index $k^{\prime}$ of this pair satisfies $k^{\prime}=\left|C_{\sigma} / \bar{G}_{\sigma}\right| \cdot j_{0}$. Similarly, $k=\left|C_{\leq \sigma} / \bar{G}\right|=\left|C_{\sigma} / \bar{G}_{\sigma}\right| \cdot j$. Since $k$ is minimal, $j \leq j_{0}$ and $j$ is minimal.

To see the last statement of the Proposition, again use 1.1(b) to obtain

$$
\left|C_{\leq \sigma} / \bar{G}\right|=\left|C_{\sigma} / \bar{G}_{\sigma}\right| \cdot\left|C_{<\sigma} / \bar{G}_{<\sigma}\right| \text {. }
$$

This implies that $\left|C_{\leq \sigma} / \bar{G}\right| \geq\left|C_{<\sigma} / \bar{G}_{<\sigma}\right|$. On the other hand, $\bar{G} \simeq \bar{G}_{\sigma} \oplus \bar{G}_{<\sigma}$ and $\bar{G}_{\sigma}$ is homogeneous completely decomposable of type $\sigma$. Thus, there is an embedding $\bar{G} \rightarrow \bar{G}_{\sigma} \oplus C_{<\sigma}$ which is an $A C D$-pair of index $\left|C_{<\sigma} / \bar{G}_{<\sigma}\right|$. Since $\left|C_{\leq \sigma} / \bar{G}\right|$ is minimal by (a), we have $\left|C_{\leq \sigma} / \bar{G}\right| \leq\left|C_{<\sigma} / \bar{G}_{<\sigma}\right|$. 
We are now ready for the main theorem of this section.

TheOREM 1.5. Let $G \subset C=\bigoplus C_{\sigma}$ be an $A C D$-pair. The following are equivalent.

(a) $|C / G|$ is minimal.

(b) If $\bar{G}_{\leq \sigma}$ and $\bar{G}_{<\sigma}$ are the projections of $G$ into $C_{\leq \sigma}$ and $C_{<\sigma}$ respectively, then $\bar{G}_{\leq \sigma}=C_{\sigma} \oplus \bar{G}_{<\sigma}$.

(c) Each $\alpha_{\sigma}^{\prime}: G^{*}[\sigma] / G[\sigma] \rightarrow C_{\sigma}$ is an isomorphism and there are splitting maps $\pi_{\sigma}: G / G[\sigma] \rightarrow G^{*}[\sigma] / G[\sigma]$ which make the following diagram commute.

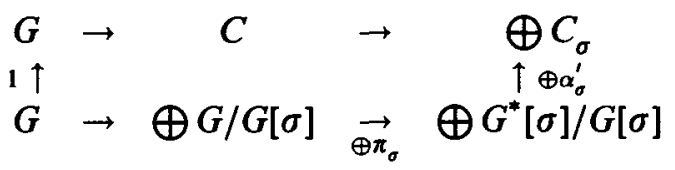

(d) $|C / G|=\mu(G)$.

Proof. (a) $\rightarrow$ (b) By Proposition 1.4, $\left|C_{<\sigma} / \bar{G}\right|=\left|C_{<\sigma} / \bar{G}_{<\sigma}\right|$. It follows that $C_{\sigma}=\bar{G}_{\sigma}$ from Proposition 1.1(b). This implies $\bar{G}=\bar{G}_{\sigma} \oplus \bar{G}_{<\sigma}=$ $\bar{C}_{\sigma} \oplus \bar{G}_{<\sigma}$ by the Modular Law.

(b) $\rightarrow$ (c) The equalities $\bar{G}=\bar{G}_{\sigma} \oplus H=C_{\sigma} \oplus \bar{G}_{<\sigma}$ (with $H \simeq G / G^{*}[\sigma]$ ) imply that $\bar{G}_{\sigma}=C_{\sigma}$, since $\operatorname{Hom}\left(C_{\sigma}, H\right)=0$. Thus, each $\alpha_{\sigma}^{\prime}$ is an isomorphism. To obtain the required splitting map $\pi_{\sigma}$, use the composition

$$
G / G[\sigma] \stackrel{\alpha_{\sigma}^{\prime}}{\longrightarrow} C_{\leq \sigma} \stackrel{\text { proj }}{\longrightarrow} C_{\sigma} \stackrel{\left(\alpha_{\sigma}^{\prime}\right)^{-1}}{\longrightarrow} G^{*}[\sigma] / G[\sigma]
$$

It is easy to check that the diagram in (c) commutes.

(c) $\rightarrow$ (a) and (d) Choose $G \subseteq D$ an $A C D$-pair of minimal index. Since we have already shown $(a) \rightarrow(b) \rightarrow(c)$, there is a commutative diagram

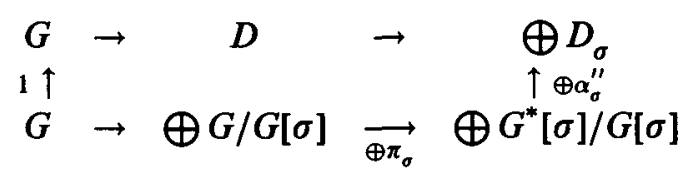

with $\bigoplus \alpha_{\sigma}^{\prime \prime}$ an isomorphism. This shows that $|D / G|=\mu(G)=|C / G|$ by Proposition 1.2. That is, $|C / G|=\mu(G)$ is minimal.

(d) $\rightarrow$ (a) If $G \subset C$ is an $A C D$-pair with $|C / G|=\mu(G)$ and $G \subset D$ is an $A C D$-pair with $|D / G|$ minimal, then by what has been shown, $|D / G|=$ $\mu(G)=|C / G|$ and $|C / G|$ is minimal.

If $G \subseteq C$ is an $A C D$-pair satisfying the conditions of Theorem 1.5, we will call $C$ a regulating hull for $G$. The next example shows that regulating hulls are not as plentiful as one might suspect. 
EXAMPLE 1.6. If $G \subset D$ is an $A C D$-pair, it need not be the case that $D$ contains a regulating hull of $G$. Let $A, B \subset S$ be subgroups of $Q$ such that $A$ and $B$ have incomparable types which are strictly less than the type of $S$. Assume $p$ is a prime for which $p^{-1} \notin S$. Form the completely decomposable group $D=A a \oplus B b \oplus S c_{1} \oplus S c_{2}$, where $a, b, c_{1}, c_{2}$ are assumed to be elements of $D$ of $p$-height 0 . Let $G$ be the subgroup of $D$ given by $G=A\left(p a+c_{1}\right) \oplus B\left(p b+c_{2}\right) \oplus p S c_{1} \oplus S\left(c_{1}+c_{2}\right)+Z(a+b)$. Finally, let $C=A\left(a-c_{2} / p\right) \oplus B\left(b+c_{2} / p\right) \oplus p S c_{1} \oplus S\left(c_{1}+c_{2}\right) \subset Q D$. The following facts will be shown.

(1) $G$ is a subgroup of $C$ and $D$.

(2) $C / G$ is cyclic of order $p$, generated by $a-c_{2} / p+G$.

(3) $D / G$ is a cyclic group of order $p^{2}$ generated by $a+G$.

(4) $G$ is not completely decomposable.

(5) $C$ is a regulating hull of $G$.

(6) $E=G+Z p a \leq D$ is not a regulating hull of $G$.

Facts (1) and (2) are routine. For (3), it is easy to check that $Z a+G=D$ and that $p^{2} a \in G$. Assuming $p a \in G$ gives an equation

$$
p a=\alpha\left(p a+c_{1}\right)+\beta\left(p b+c_{2}\right)+\gamma p c_{1}+\gamma^{\prime}\left(c_{1}+c_{2}\right)+n(a+b),
$$

with $\alpha \in A, \beta \in B, \gamma, \gamma^{\prime} \in S$ and $n \in Z$. Solving gives $\gamma=-1 / p \in S$, contrary to hypothesis. To see (4), suppose $G$ is completely decomposable. Since the type of $S$ is a maximum, $G=X \oplus H$, where $H=p S c_{1} \oplus S\left(c_{1}+c_{2}\right)$ and $X \simeq A \oplus B$. But it is easy to check that $X \simeq G / H \simeq A p a \oplus B p b+Z(a+$ $b$ ), and the last group is indecomposable. This contradiction completes the proof of (4).

It follows from (4) that $C$ is a regulating hull of $G$, since $C$ is completely decomposable and $|C / G|=p$ is minimal. This gives (5). Moreover, $D$ contains a unique proper subgroup which contains $G$, namely, the subgroup $E$ generated by $G$ and $p a$. In particular, $E$ contains $c_{1}=p a+c_{1}-p a$ and $p S c_{1}$, so $E$ contains $S c_{1}$ and therefore $S c_{2}$. Thus, if $\sigma=\operatorname{type}(S)$, then $E_{\sigma}=S c_{1} \oplus S c_{2}$ is a summand of $E$, while $G^{*}[\sigma]=G^{*}[\sigma] / G[\sigma]=$ $p S c_{1} \oplus S c_{2}$. By Theorem 1.5(c), $E$ cannot be a regulating hull for $G$, which is (6). Since $E$ is the unique candidate for a regulating hull inside $D$, we have completed the proof of the example. Note that $E$ cannot be completely decomposable, and that $D$ is therefore a regulating hull for $E$. Also note that $G$ projects onto each homogeneous component of $D$. Thus, this condition is not sufficient to imply that $D$ is a regulating hull for $G$, although it is clearly necessary.

As mentioned in the introduction, Burkhardt [5] introduced the regulator of an almost completely decomposable group and established some important 
properties of it. Our next theorem provides the analogous results on the "dual" of the regulator. Since the regulator is the intersection of the regulating subgroups, the coregulator will be the sum of the regulating hulls, taken in an appropriate vector space over $Q$.

Theorem 1.7. Let $C=\bigoplus C_{\sigma}$ be a regulating hull for $G$, and denote $E(G)=\sum\{D: D$ a regulating hull with $Q D=Q C\}$. Then $E(G)=$ $\bigoplus d_{\sigma}^{-1} C_{\sigma}$, where $d_{\sigma}=\operatorname{lcm}\left\{\right.$ exponent $D_{<\sigma} / \bar{G}_{<\sigma}: D$ is a regulating hull of $G$. Moreover, any endomorphism of $G$ induces a unique endomorphism of $E(G)$.

Proof. We first show $E(G) \subseteq \bigoplus d_{\sigma}^{-1} C_{\sigma}$. Let $D$ be a regulating hull with $Q D=Q C$. By definition of $d_{\sigma}$ and Theorem 1.5, $d_{\sigma} D_{\leq \sigma} \subseteq G+D[\sigma]$. Projection onto $C_{\sigma}$ gives image $d_{\sigma} D_{\leq \sigma} \subseteq$ image $G=C_{\sigma}$. In other words, image $D_{\leq \sigma} \subseteq d_{\sigma}^{-1} C_{\sigma}$. But $D_{\leq \sigma}$ is the only part of $D$ which can map into $C_{\sigma}$. It follows that $D \subseteq \bigoplus d_{\sigma}^{-1} C_{\sigma}$.

One more lemma allows us to complete the proof of Theorem 1.7.

LEMma 1.8. If $C$ is a regulating hull of $G$ and the $A C D$-pair $\beta_{\sigma}: G / G[\sigma]$ $\rightarrow C_{<\sigma}$ has index $p^{m} k$ with $(k, p)=1$, then $p^{-m} C_{\sigma} \subseteq E(G)$.

Proof. Refer to Proposition 1.1(b) for the definition of $\beta_{\sigma}$. By hypothesis there exists $a \in C_{<\sigma}$ with order $(a)=p^{m}$ modulo $\bar{G}_{<\sigma}=\beta_{\sigma}\left(G / G^{*}[\sigma]\right)$. It is immediate that $p$-height $(a)=0$ in $C_{<\sigma}$ and $p$-height $\left(p^{m} a\right)=0$ in $\bar{G}_{<\sigma}$. Each rank one factor of $\bar{G}_{<\sigma}$ has type $\leq \sigma$. If $0 \neq x \in C_{\sigma}$, then by Remark (2) on page 108 of [2], there is a homomorphism $f: \bar{G}_{<\sigma} \rightarrow\langle x\rangle_{*} \subseteq C_{\sigma}$ such that $f\left(p^{m} a\right)=t x$, where $t$ is a nonzero integer prime to $p$. Further,

$$
C_{\leq \sigma}=C_{\sigma} \oplus C_{<\sigma} \simeq C_{\sigma} \oplus(1+f) C_{<\sigma} \supset C_{\sigma} \oplus(1+f) \bar{G}_{<\sigma}=C_{\sigma} \oplus \bar{G}_{<\sigma}=\bar{G}_{\leq \sigma} .
$$

Thus, $D=C_{\sigma} \oplus(1+f) C_{<\sigma} \oplus C[\sigma]$ is a regulating hull for $G$. Moreover, $t p^{-m} x=f(a)=(1+f) a-a \in D+C$, whence $p^{-m} x \in D+C \subset E(G)$ since $(t, p)=1$. It follows that $p^{-m} C_{\sigma} \subseteq E(G)$ and the proof of the lemma is complete.

Returning to the proof of Theorem 1.7, we show $d_{\sigma}^{-1} C_{\sigma} \subseteq E(G)$ for every regulating hull $C=\oplus C_{\sigma}$ of $G$. Again it suffices to fix a prime $p$ and show $p^{-m} C_{\sigma} \subset E(G)$, where $m=$ p-height $\left(d_{\sigma}\right)$. Choose a regulating hull $D$ of $G$ so that $p^{m}$ divides the index of $G / G^{*}[\sigma] \rightarrow D_{<\sigma}$. By Lemma 1.3, we can assume $Q D_{\leq \sigma}=Q C_{\leq \sigma}$ and form a new regulating hull $D^{\prime}=D_{\leq \sigma} \oplus C[\sigma]$ of 
$G$. Write $0 \neq x \in C_{\sigma}$ as $x=x_{1}+x_{2}$ with $x_{1} \in D_{\leq \sigma}$ and $x_{2} \in C[\sigma]$. First, $x_{1} \in D_{\sigma}$ since type $(x)=\sigma$. Thus, $p^{-m} x_{1} \in E(G)$ by Lemma 1.8. Second, $x_{2} \in C[\sigma]$ has type $\left(x_{2}\right) \geq \sigma$. This implies $x_{2} \in C^{*}(\sigma)=\sum_{\tau>\sigma} C_{\tau}$. If $\sigma$ is a maximal type, then $C^{*}(\sigma)=0$ and $p^{-m} x=p^{-m} x_{1} \in E(G)$. If $\sigma$ is not maximal, write $x_{2}=\sum x_{\tau}$, where $x_{\tau} \in C_{\tau}$ with $\tau>\sigma$. By induction on the type lattice, $d_{\tau}^{-1} x_{\tau} \in E(G)$ for $\tau>\sigma$. However, $\tau>\sigma$ implies $d_{\sigma}$ divides $d_{\tau}$, by the definition of $d_{\sigma}$. This establishes that $p^{-m} x_{2} \in E(G)$, so that $p^{-m} x \in E(G)$ and $E(G)=\bigoplus d_{\sigma}^{-1} C_{\sigma}$ as desired.

To prove the final statement of 1.7 , let $\theta: G \rightarrow G$ be an endomorphism. Take $C$ and $D$ to be regulating hulls of $G$ and $E(G)=\bigoplus d_{\sigma}^{-1} C_{\sigma}$, as above. If $\gamma_{\sigma}$ denotes the vector space projection $Q C \rightarrow Q C_{\sigma}$, then $\gamma_{\sigma} \theta\left(d_{\sigma} D\right)=$ $\gamma_{\sigma} \theta\left(d_{\sigma} D_{\leq \sigma}\right) \subseteq \gamma_{\sigma} \theta(G)$, the last containment by definition of $d_{\sigma}$. But $\gamma_{\sigma} \theta(G)$ $\subseteq \gamma_{\sigma}(G)=C_{\sigma}$, since $C$ is a regulating hull of $G$. We may conclude that $\theta(D) \subseteq \bigoplus d_{\sigma}^{-1} C_{\sigma}=E(G)$ for each regulating hull $D$ of $G$. It follows that $\theta(E(G))=\theta\left(\sum\right.$ regulating hulls of $\left.G\right) \subseteq E(G)$. This completes the proof.

\section{Warfield duality}

In this section we employ a duality discovered by Warfield [11] to show that our results are equivalent to those of Lady and Burkhardt in certain contexts, namely, those where the critical typeset is locally free. We begin by recalling the relevant definitions and results.

Let $X$ be a subgroup of $Q$. A torsion-free group $G$, always of finite rank, is $X$-locally free provided outer type $(G) \leq \operatorname{type}(X)$ and $p X=X$ if and only if $p G=G$. Warfield showed, in essence, that $\operatorname{Hom}(-, X)$ provides a duality from the category of $X$-locally free groups and homomorphisms to itself. Our interest in this duality is that it does not require passing to a "quasi" category. We can apply it to groups which are quasi-equal without losing information. We will denote $W G=\operatorname{Hom}(G, X)$ whenever it is clear what $X$ is; and if $\sigma$ is the type of a subgroup $A$ of $X$, then $\sigma^{w}$ is the type of $\operatorname{Hom}(A, X)$.

We begin with a housekeeping lemma.

LEMMA 2.1. Let $X$ be a subgroup of $Q$.

(a) If $G \subseteq C$ is an $A C D$-pair of $X$-locally free groups, then there is an inclusion induced by restriction, $\operatorname{Hom}(C, X) \subseteq \operatorname{Hom}(G, X)$, and $C / G \simeq$ $\operatorname{Hom}(G, X) / \operatorname{Hom}(C, X)$ as groups.

(b) If $A$ and $B$ are rank one $X$-locally free groups, then type $(A) \leq \operatorname{type}(B)$ if and only if $\operatorname{type}(\operatorname{Hom}(B, X)) \leq \operatorname{type}(\operatorname{Hom}(A, X))$, with equality holding either in both cases or in neither case. 
Proof. The induced containment in part (a) is routine. Moreover, both $C / G$ and $\operatorname{Hom}(G, X) / \operatorname{Hom}(C, X)$ are finite groups, so it suffices to show that their $p$-components are isomorphic. If $(C / G)_{p}$ is non-zero, then $p C \neq$ $C$, so that $G, C$ and $X$ are $p$-reduced by the definition of $X$-locally free. In particular, $G_{p}$ and $C_{p}$ are free $Z_{p}$-modules for which there is a stacked basis. Thus, to show $(C / G)_{p}=C_{p} / G_{p}$ is isomorphic to $\operatorname{Hom}(G / X)_{p} / \operatorname{Hom}(C, X)_{p}$ $=\operatorname{Hom}\left(G_{p}, X_{p}\right) / \operatorname{Hom}\left(C_{p}, X_{p}\right)$, it suffices to consider the case in which rank $G_{p}=\operatorname{rank} C_{p}=1$. In this case the result is immediate.

Part (b) is a consequence of the fact that $\sup \{\operatorname{type}(A), \operatorname{type}(\operatorname{Hom}(A, X))\}$ $=\operatorname{type}(X)$ when $A$ is an $X$-locally free subgroup of $X$.

Following Lady [7], we denote, for any torsion-free group $G$ and type $\sigma, G^{\#}(\sigma)=\left(\sum_{\tau>\sigma} G(\tau)\right)_{*}$, the pure subgroup generated by the elements of type bigger than $\sigma$; and $G(\sigma)=\{x \in G$ : type $(x) \geq \sigma\}$, a pure subgroup containing $G^{\#}(\sigma)$.

THEOREM 2.2. Let $X$ be a subgroup of $Q, G$ an $X$-locally free Butler group and $\sigma$ an element of typeset $(G)$. Then there are commutative diagrams with exact rows and vertical isomorphisms,

(a)

$$
\begin{array}{ccccccc}
0 \rightarrow W(G / G[\sigma]) & \rightarrow & W G & \rightarrow & W(G[\sigma]) & \rightarrow 0 \\
& \downarrow \alpha & & \downarrow 1 & & \downarrow & \\
0 \rightarrow & W G\left(\sigma^{w}\right) & \rightarrow & W G & \rightarrow & W G / W G\left(\sigma^{w}\right) & \rightarrow 0
\end{array}
$$

(b)

$$
\begin{aligned}
& 0 \rightarrow W\left(G / G^{*}[\sigma]\right) \rightarrow W G \rightarrow W\left(G^{*}[\sigma]\right) \quad \rightarrow 0
\end{aligned}
$$

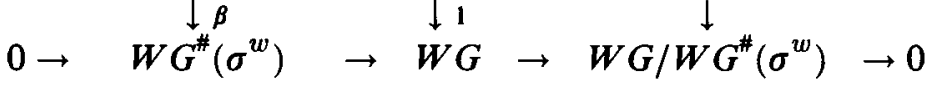

(c)

$$
\begin{aligned}
& 0 \rightarrow W\left(G / G^{*}[\sigma]\right) \rightarrow W(G / G[\sigma]) \rightarrow W\left(G^{*}[\sigma] / G[\sigma]\right) \quad \rightarrow 0 \\
& \downarrow \beta \quad \downarrow \alpha \\
& 0 \rightarrow W G^{\#}\left(\sigma^{w}\right) \quad \rightarrow W G\left(\sigma^{w}\right) \rightarrow W G\left(\sigma^{w}\right) / W G^{\#}\left(\sigma^{w}\right) \rightarrow 0
\end{aligned}
$$

Proof. The theorem shows what happens when we dualize the exact sequences induced by the $\sigma$-socle and the $\sigma$-radical of $G$. For example, (a) describes the dual of the sequence $0 \rightarrow G[\sigma] \rightarrow G \rightarrow G / G[\sigma] \rightarrow 0$. Note that by replacing $W G$ by $G$ and $\sigma^{w}$ by $\sigma$, then dualizing, we obtain a description of the dual of $0 \rightarrow G(\sigma) \rightarrow G \rightarrow G / G(\sigma) \rightarrow 0$. We will give the proof of $(b)$, the others being similar (see also $[3,9,10]$ ). 
Because the sequence $0 \rightarrow G^{*}[\sigma] \rightarrow G \rightarrow G / G^{*}[\sigma] \rightarrow 0$ is pure exact, applying $\operatorname{Hom}(-, X)$ yields an exact sequence $0 \rightarrow W\left(G / G^{*}[\sigma]\right) \rightarrow W G \rightarrow$ $W\left(G^{*}[\sigma]\right) \rightarrow 0$ because $X$ is pure injective in the category of Butler groups with typeset bounded above by $\operatorname{type}(X)$ (see [8] or [2; Prop. 1.6]). We show that the image of $W\left(G / G^{*}[\sigma]\right)$ in $W G$ is $W G^{*}\left(\sigma^{w}\right)$. Statement (b) is then an immediate consequence. Let $A$ be a subgroup of $X$ with type $(A)<\sigma$, so that $\sigma^{w}<\operatorname{type}(\operatorname{Hom}(A, X))$. If $0 \neq f: G / G^{*}[\sigma] \rightarrow A$ is an element of $W\left(G / G^{*}[\sigma]\right)$, then the image of $f$ in $W G$ is the composition of $f$ with the quotient map $G \rightarrow G / G^{*}[\sigma]$. If $K$ is the kernel of $f$, then the type of $f$ in $W G$ is the type of the rank one group $\operatorname{Hom}(G / K, X)$, and $G / K$ is $X$-locally free. But $\operatorname{type}(G / K) \leq \operatorname{type}(A)$, so $\sigma^{w}<\operatorname{type}(\operatorname{Hom}(A, X)) \leq$ $\operatorname{type}(\operatorname{Hom}(G / K, X))=\operatorname{type}(f)$. It follows that $f \in W G\left(\sigma^{w}\right)$. The definition of $G^{*}[\sigma]$ implies that $W\left(G / G^{*}[\sigma]\right)=\operatorname{Hom}\left(G / G^{*}[\sigma], X\right)$ contains a maximal linearly independent set of maps of the form $f: G / G^{*}[\sigma] \rightarrow A$ with type $(A)<\sigma$. We may conclude that the image of $W\left(G / G^{*}[\sigma]\right)$ is contained in $W G^{*}\left(\sigma^{w}\right)$. The reverse inclusion is essentially a reverse argument. Let $f \in W G^{*}\left(\sigma^{w}\right)$ have type greater than $\sigma^{w}$. If $K$ is the kernel of $f$, then by Lemma $2.1, \operatorname{type}(G / K)<\sigma$. Thus, $G^{*}[\sigma] \subseteq K$, and $f$ is in the image of $W\left(G / G^{*}[\sigma]\right)$.

We can now apply Warfield duality to the results of Section 1.

Proposition 2.3. Let $G$ be an almost completely decomposable group. For any choice of splitting maps $\rho_{\tau}: G(\tau) / G^{*}(\tau) \rightarrow G(\tau)$, the composition $\bigoplus G(\tau) / G^{\#}(\tau) \rightarrow \bigoplus G(\tau) \rightarrow G$ defines an $A C D$-pair. The index $\varphi(G)$ of this pair is independent of the choice of the $\rho_{\tau}$.

Theorem 2.4. Let $\oplus C_{\tau}=C \subset G$ be a female ACD-pair. The following are equivalent.

(a) $|G / C|$ is minimal.

(b) For each $\tau, G(\tau)=C_{\tau} \oplus G^{\#}(\tau)$.

(c) Each composition $\gamma_{\tau}: C_{\tau} \rightarrow G(\tau) \rightarrow G(\tau) / G^{*}(\tau)$ is an isomorphism and there are splitting maps $\rho_{\tau}: G(\tau) / G^{*}(\tau) \rightarrow G(\tau)$ such that the following diagram commutes.

(d) $|G / C|=\varphi(G)$.

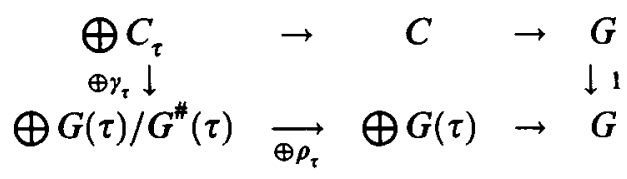

These two results can either be found in, or derived from results in [7]. 
In the locally free setting, they are direct dualizations of Proposition 1.2 and Theorem 1.5. The duality in conditions $1.5(\mathrm{~b})$ and $2.4(\mathrm{~b})$ requires only the observation that $2.4(\mathrm{~b})$ (respectively, $1.5(\mathrm{~b})$ ) is equivalent to the statement that each $\gamma_{\tau}$ (respectively, $\alpha_{\sigma}^{\prime}$ ) is an isomorphism.

We conclude with Burkhardt's result [5], which is a direct dualization of Theorem 1.7 in the locally free setting.

TheOREM 2.5. Let $C \subseteq G$ be a female $A C D$-pair with $C$ a regulating subgroup of $G$. Denote

$$
R(G)=\bigcap\{D: D \text { a regulating subgroup of } G\}
$$

Then $R(G)=\bigoplus d_{\tau} C_{\tau}$, where $d_{\tau}=\operatorname{lcm}\left\{\exp \left(G^{*}(\tau) / D^{*}(\tau)\right): D\right.$ a regulating subgroup of $G\}$. Moreover, $R(G)$ is a fully invariant subgroup of $G$.

\section{References}

[1] D. Arnold, 'Pure subgroups of completely decomposable groups', in: Abelian Group Theory Proceedings, Oberwolfach 1981, Lecture Notes in Mathematics 874, (Springer, Berlin, 1981) pp. 1-31.

[2] D. Arnold and C. Vinsonhaler, 'Pure subgroups of finite rank completely completely decomposable groups, II', in Abelian Group Theory, Proceedings, Honolulu 1982/83, Lecture Notes in Mathematics 1006 (Springer, Berlin, 1983) pp. 97-143.

[3] __, 'Duality and invariants for Butler groups', Pacific J. Math. 148 (1991), 1-10.

[4] R. Baer, 'Abelian groups without elements of finite order', Duke Math. J. 3 (1937), 68-122.

[5] R. Burkhardt, 'On a special class of almost completely decomposable groups', in: Abelian Groups and Modules, Proceedings of the Udine Conference 1984, CISM 287 (Springer, New York, 1984), pp. 141-150.

[6] K. J. Krapf and O. Mutzbauer, 'Classification of almost completely decomposable groups', in: Abelian Groups and Modules, Proceedings of the Udine Conference 1984, CISM 287 (Springer, New York 1984), 151-162.

[7] E. L. Lady, 'Almost completely decomposable torsion-free groups', Proc. Amer. Math. Soc. 45 (1974), 41-47.

[8] __ , 'Extension of scalars for torsion free modules over Dedekind domains', in: Symposia Mathematica Vol. XXIII (Academic Press, San Diego, 1979), pp. 287-305.

[9] _ 'Warfield duality and rank-one quasi-summands of tensor products of finite rank locally free modules over Dedekind domains', J. Algebra 121 (1989), 129-138.

[10] J. D. Reid, 'Warfield duality and Irreducible Groups', preprint (to appear in Warfield Commemorative Volume). 
[11] R. B. Warfield, Jr., 'Homomorphisms and duality for torsion-free groups', Math Z. 107 (1968), 189-200.

University of Hawaii

Honolulu, HI 96822

USA
University of Connecticut

Storrs, CT 06269

USA 